\title{
Influencia de la deuda en la estrategia de exportación de la empresa familiar
}

\section{Influence of the debt on the export strategy of family firms}

\author{
Cristina López-Cózar Navarro $^{\mathrm{a}^{*}} \cdot$ Tiziana Priede Bergamini $^{\mathrm{b}} \cdot$ Sonia Benito Hernández $^{\mathrm{c}}$ \\ ${ }^{a}$ Departamento de Economía y Ciencias Sociales Agrarias, Universidad Politécnica de Madrid, Madrid (Spain) \\ ${ }^{b}$ Departamento de Economía y Relaciones Internacionales, Universidad Europea de Madrid, Madrid (Spain) \\ ${ }^{c}$ Departamento de Estadística y Métodos de Gestión, Universidad Politécnica de Madrid, Madrid (Spain)
}

D A T OS ARTÍC ULO

\section{Historial:}

Recibido 09-02-2013

Aceptado 01-05-2013

Palabras clave: Empresa familiar

Exportación

Endeudamiento

Códigos JEL:

G32, F20, M10

\section{A R T I C LE INF O}

Article history:

Received 09-02-2013

Accepted 01-05-2013

Keywords:

Family Firm

Debt

Export

JEL codes:

G32, F20, M10

\section{R E S U M E N}

Este trabajo estudia si el carácter familiar de la empresa influye a la hora de tomar la decisión de emprender una estrategia de exportación y el papel que desempeña el nivel de endeudamiento ante esta decisión.

Para ello, se ha realizado una revisión de la literatura y un estudio empírico con una muestra de 1.846 empresas industriales españolas, entre las que se encuentran familiares y no familiares. Los resultados parecen demostrar que la propiedad familiar no es un factor determinante en la decisión de exportar sus productos, sin embargo, la importancia del endeudamiento difiere entre las empresas familiares y aquéllas que no lo son.

\section{A B S T R A C T}

This paper studies whether the family character of the company has an influence when deciding to start up an export strategy and the relevance of debt when facing this decision.

To do so, a review of the literature on the subject has been developed and an empirical analysis using a sample of 1.846 businesses, which include both family and non-family firms. The results seem to show that family character is not a determinant factor when deciding to export, nevertheless, the importance of debt differs among family and non-family firms.

* Autor de contacto.

Correoselectrónicos: cristina.lopezcozar@upm.es,tiziana.priede@uem.es,sonia.benito@upm.es 


\section{Introducción}

El fenómeno de la internacionalización ha sido ampliamente estudiado en la literatura, sin embargo, no existen muchos trabajos dedicados al caso particular de las empresas familiares. Una de las principales características de este tipo de empresas es la concurrencia de tres grupos de personas: los miembros de la familia, los propietarios de la empresa y los directivos de la misma, cada uno con su propio sistema de valores y objetivos, lo que implica la existencia de múltiples y complejas relaciones entre ellos (López-Cózar y Priede, 2009). Precisamente, estas interrelaciones provocan que su comportamiento sea diferente al de otras empresas. En este sentido, diversos autores coinciden en señalar que el proceso de establecimiento de los objetivos estratégicos y de los planes para alcanzarlos, no es igual que en las empresas no familiares, debido a la gran influencia que ejerce el interés particular de la familia que controla la empresa (Martín y Cabrera, 2007) y, aunque existen excepciones, el crecimiento no suele ser un objetivo prioritario para estas organizaciones (Claver et al., 2009).

En la misma línea, distintos estudios coinciden en señalar que la actividad internacional de las empresas familiares es menor que en el caso de empresas no familiares (Gallo y García-Pont, 1996; Okoroafo, 1999; Davis y Harveston, 2000; Acedo et al., 2004; Graves y Thomas, 2004; Menendez, 2004; Fernández y Nieto, 2005, 2006; Sacristan et al., 2011). Así, Gallo y Sveen (1991) sugieren que el proceso de internacionalización en las empresas familiares es más lento que en las no familiares, $\mathrm{y}$ que en general, suelen concentrarse en atender las necesidades de los mercados domésticos, salvo aquellos casos en los que los directivos tengan experiencia internacional personal (realización de viajes, conocimiento de idiomas, contactos o familiares en otros países, etc.).

Por su parte, Claver et al. (2009) destacan que las empresas familiares presentan una actividad internacional similar a las no familiares (medida tanto por la propensión exportadora, como a través del número de inversiones directas en el exterior). Mientras otros estudios como el de Sacristán et al. (2011) demuestran que, en el caso de pequeñas y medianas empresas, sí existen ciertas diferencias en la propensión exportadora entre las familiares y las que no lo son, mostrando las primeras valores muy inferiores a los obtenidos para las pymes, las cuales, por su parte, pueden seguir los patrones de los procesos incrementales de internacionalización o modelo relacionado con la innovación (I-Model), pero no así las familiares.

En general, las empresas familiares cuentan con algunas peculiaridades que pueden considerarse como puntos fuertes a la hora de llevar a cabo dicho proceso de internacionalización. Entre éstos, destaca la experiencia y conocimiento del negocio, del producto y del propio sector; así como, una cultura corporativa fuerte, con convicciones y valores compartidos y un alto sentimiento de pertenencia al grupo. En este sentido, la confianza, la lealtad y el compromiso de sus miembros son superiores al de otras empresas, lo que hace que se muestren más fuertes ante las situaciones complejas (Swinth y Vinton, 1993; Lansberg 1999; Okoroafo, 1999; Habbershon y Williams, 2000; Karlsson, 2001; Fuentes et al., 2007 a; Gallo et al., 2009).

No obstante, también existen otros aspectos que pueden suponer una limitación a la actividad internacional, como son los problemas que pueden surgir en el relevo generacional, así como la falta de profesionalización y estructuras organizativas poco claras e informales (Gallo y Sveen, 1991; Gallo y García-Pont, 1996; Graves y Thomas, 2004; Fuentes et al., 2007 a). De hecho, la estrategia de internacionalización es una de las que en mayor medida requiere el máximo nivel de profesionalización (Quintana, 2007). Así mismo, diferentes autores señalan la falta de recursos financieros (Barry, 1975; Luostarinen y Hellamn, 1994; Gallo y GarcíaPont, 1996; Fuentes et al., 2007 b; Claver et al., 2009) y en general, la dificultad de acceder a los recursos de diferente índole, tanto tangibles como intangibles, necesarios para emprender dicha actividad, así como su aversión al riesgo y espíritu más conservador, al estar el sustento de 
la familia unido al negocio (Fernández y Nieto, 2005).

Dentro de la empresa familiar, pueden encontrarse importantes diferencias entre las de primera generación, segunda y ulteriores. En este sentido, Okoroafo (1999) sugiere que aquellas empresas que no han llevado a cabo un proceso de internacionalización en la primera o en la segunda generación, es más difícil que lo hagan más adelante. En la misma línea, Westhead et al. (2001), consideran que es más probable que la primera generación sea más proclive a la actividad exportadora, porque tienen más recursos y conocimientos. Sin embargo, otros trabajos apuntan justamente lo contrario; algunos estudios ponen de manifiesto que a partir de la segunda generación es más fácil que la empresa comience un proceso de internacionalización (Gallo y García Pont, 1996; Fernández y Nieto, 2005). Por su parte, Claver et al (2009) no encuentran evidencia de que la segunda y siguientes generaciones realicen una mayor inversión en el exterior. Más adelante, Okoroafo y Perryy (2010) confirman que la segunda generación es más receptiva a emprender un proceso de internacionalización.

Otros estudios se centran en las capacidades, las actitudes o las características personales del director general (en inglés CEO) (Gallo y García Pont, 1996; Davis y Harveston, 2000; Graves y Thomas, 2004; Casillas y Acedo, 2005), como su nivel de formación o su edad. Por ejemplo, Davis y Harveston (2000) llegan a la conclusión de que existe una relación positiva entre la formación del directivo y el grado de internacionalización de la empresa, pero sin embargo, su edad no supone diferencias en la propensión a exportar.

El presente trabajo tiene como objetivo estudiar qué factores influyen en la decisión de exportación de la empresa familiar, haciendo especial hincapié en el nivel de endeudamiento. Para alcanzar dicho objetivo, el trabajo realiza una revisión bibliográfica sobre los factores que influyen en la estrategia de exportación de la empresa familiar, así como un estudio empírico basado en los datos obtenidos en la Encuesta de Estrategias Empresariales (realizada por el Ministerio de Ciencia y Tecnología y la
Fundación SEPI) de 1.846 empresas industriales (familiares y no familiares), pertenecientes al sector industrial español. El trabajo concluye con unas reflexiones finales a partir de los resultados obtenidos en el contraste empírico.

\section{Revisión de la literatura}

Desde un punto de vista genérico, existen básicamente dos opciones para llevar a cabo la penetración en mercados exteriores: desde el mercado de origen, mediante una estrategia de exportación, o bien haciendo inversiones directas en el país de destino. A su vez, estas dos opciones pueden llevarse a cabo de manera individual $o$ formalizando acuerdos de cooperación con otras empresas (Fuentes y Fernandez, 2010). En general, la elección de una alternativa $u$ otra depende tanto de factores internos de la empresa, como el tamaño, la experiencia internacional o la pertenencia de la empresa a grupo empresarial; como de factores del sector industrial, como el grado de concentración o la existencia de clientes internacionales en la industria; y de factores relacionados con el país de destino, como el tamaño del mercado o el riesgo (Martín, 2004).

Según el modelo escadinavo propuesto por distintos autores de la escuela de Uppsala ( $U$ Model) (ie., Johanson y Wiedershein, 1975; Luostarinem, 1970; Johanson y Vahlne, 1977; Johanson y Vahlne, 1990; Vahlne y Nordström, 1993), conocido como el paradigma de las etapas de desarrollo internacional, se puede entender la internacionalización de la empresa como un proceso incremental que se va desarrollando a lo largo del tiempo, según se van obteniendo conocimientos y experiencia en los nuevos mercados. De esta manera, las empresas suelen empezar su aventura internacional con una estrategia de exportación, ya que esta opción suele revestir menos riesgo y menores costes, si bien en algunos casos, producir directamente en el país de destino pude resultar más económico (economía de localización, costes de transporte, etc.). Por su parte, con aquellas estrategias de internacionalización que implican inversiones en el exterior, ya sea estableciendo una joint 
venture o mediante una subsidiaria propia, la empresa puede ejercer un mayor control sobre todo el proceso, pero lleva aparejados altos riesgos y costes, especialmente en el caso de filiales de pleno dominio (Hill y Kim, 1990). Por este motivo, las pequeñas empresas suelen preferir la exportación frente a las inversiones directas en el país de destino (Pla-Barber, 2001).

Respecto a las empresas familiares, la literatura demuestra que parece existir un cierto consenso en encontrar una relación negativa entre propiedad familiar e internacionalización, y más aún si son empresas de pequeña dimensión (Fernández y Nieto, 2002; Gallo y García-Pont, 1996; Okorafo, 1999, Graves, 2008). Así pues, de forma global, puede afirmarse que el nivel de internacionalización de las pymes familiares españolas es bajo, desarrollado fundamentalmente a través de estrategias con poco riesgo como las exportaciones. En este sentido, Luostarinen y Hellamn (1994), encuentran que este tipo de empresas suelen tener un menor número de filiales en terceros países que las empresas no familiares.

En un reciente estudio empírico publicado para empresas industriales y de servicios (Miller et al., 2010) se pone de manifiesto que los propietarios y directivos de empresas familiares, tienden a elegir estrategias más conservadoras que las no familiares. Es decir, tienden a minimizar las inversiones en investigación y desarrollo, los gastos de capital y el endeudamiento, y prefieren desarrollar políticas de dividendos más generosas. De esta forma, sienten que minimizan los riesgos y los accionistas se sienten más seguros. En este mismo sentido, se situarían las estrategias de internacionalización, las cuales requieren de esfuerzo y capital para poder llevarse a cabo. En esta misma línea de pensamiento, el estudio realizado por Ling Lin y Fung Wu (2010) para las empresas del sector financiero, pone de manifiesto la relación negativa entre propiedad familiar y la asunción de riesgos. En este sentido se propone la siguiente hipótesis:

Hipótesis 1: Existe una relación negativa entre el carácter familiar de la empresa y su decisión de exportar.
Con respecto al nivel de endeudamiento y estructura de capital para financiar las inversiones y los procesos de internacionalización, existen múltiples estudios al respecto. La teoría de la jerarquización financiera o pecking order (Myers y Majluf, 1984; Romano et al., 2000 y Poutziouris, 2001) postula que las empresas establecen un orden de jerarquía a la hora de tomar sus decisiones de financiación. La literatura acerca de las preferencias financieras de las empresas familiares, concluye que existe gran consenso entre los autores en que dichas empresas prefieren, en primer lugar, la autofinanciación y la reinversión de beneficios a cualquier otra fuente de financiación (Corbetta, 1995; Poutziouris, 2001). En lo que no hay un claro consenso es en su preferencia por el endeudamiento.

En el caso de las empresas familiares, diferentes estudios demuestran que prefieren acudir al endeudamiento antes que a las ampliaciones de capital para financiar sus inversiones, con el fin de no dar entrada en el capital a nuevos accionistas no familiares (Chaganti y Damanpour, 1991; Hutchinson, 1995; Anderson et al., 2003). Mientras que otros autores demuestran que las empresas familiares prefieren no endeudarse y ser prudentes para evitar así perder su independencia ante los acreedores y mantener el control en caso de crisis financieras (Gallo et al, 2004; LópezGracia y Aybar-Arias, 2000).

En esta última línea, Aronoff y Astrachan (1996) explican la relación entre crecimiento y financiación mediante deuda en las empresas familiares. Tras analizar diversos estudios de empresas familiares en Estados Unidos, concluyen que en la mayor parte de los casos, éstas prefieren no endeudarse para no asumir los riesgos que ello supone, pese a las limitaciones que esta decisión tiene sobre el crecimiento de la empresa, y en consecuencia, sobre la estrategia de internacionalización. Al parecer, las empresas familiares establecen que el crecimiento, la deuda y los riesgos incrementan la complejidad de las relaciones familiares y prefieren mantener la empresa en unas dimensiones más fácilmente controlables. Los 
autores sugieren que las empresas familiares deben encontrar el equilibrio entre fuentes propias y ajenas que les permita crecer $\mathrm{y}$ maximizar su rendimiento, y al mismo tiempo, planificar y comunicar correctamente dichas estrategias a través de los órganos de gobierno.

Años más tarde, Graves y Thomas (2008) reconocen la importancia de los procesos de internacionalización para fomentar el crecimiento de las empresas familiares y analizan los principales factores que determinan dichos procesos a través de un estudio empírico, llegando a interesantes conclusiones. En primer lugar, que las empresas familiares inician procesos de internacionalización tradicionales y que los principales factores determinantes son: el nivel de compromiso de la dirección, la disponibilidad de recursos financieros y la capacidad de comprometer esos recursos y desarrollar las capacidades necesarias para la internacionalización. Dado que las empresas familiares tienen preferencias especiales en cuanto a la privacidad y el control, prefieren políticas de financiación internas favoreciendo la reinversión de los fondos propios a las ampliaciones de capital o la deuda a largo plazo, lo cual determina, como es lógico, sus políticas tradicionales de internacionalización. Esta preferencia jerárquica en la elección de las fuentes de financiación de las empresas familiares coincide con la literatura (Gallo et al, 2004; Poitziouris, 2001).

Según Blanco et al. (2009), la aspiración de perpetuar el control familiar a lo largo del tiempo, elemento clave de su definición, lleva a este tipo de empresas de forma más intensa a seguir esta política de financiación (que coincide con la citada teoría de la jerarquización), dando prioridad a la financiación interna y, en el caso de que ésta no sea suficiente, acudiendo al endeudamiento, dejando la ampliación de capital como último recurso. Al parecer, este resultado se relaciona con la generación en la que la empresa familiar se encuentra, lo cual no había sido tenido en cuenta por los trabajos anteriores y de ahí los diferentes resultados referidos al endeudamiento. En efecto, en las primeras generaciones es la unidad familiar la que gobierna y toma las decisiones financieras basadas en el sacrificio y el ahorro, de ahí que la mayoría de las inversiones sean financiadas con recursos propios $\mathrm{y}$ beneficios retenidos. A medida que la empresa y su gobierno evolucionan y se pasa a la siguiente generación, disminuyen los lazos familiares, el compromiso y la capacidad de sacrificio. No obstante, las decisiones de financiación se basan en el incremento del endeudamiento antes que en las ampliaciones de capital, por su gran aversión a la pérdida del control familiar y porque el endeudamiento resulta más barato que los recursos propios.

Por su parte, Sonfield y Lussier (2004) realizan un estudio en el que comparan diversos aspectos de la empresa familiar entre generaciones. Uno de los principales resultados obtenidos es la clara preferencia de la primera generación por el uso de capital propio respecto a las siguientes generaciones, demostrando que el uso de deuda se generaliza a medida que pasa el tiempo, cambian las generaciones y la empresa evoluciona. Estos mismos autores (Lussier y Sonfield, 2009), en un estudio posterior realizado con datos de seis países sobre la influencia del fundador en las estrategias del negocio, ponen de manifiesto que existe una correlación positiva significativa entre la influencia del fundador y el uso de capital propio, en lugar de deuda, para financiar las inversiones. En definitiva, las empresas familiares son reacias a realizar ampliaciones de capital si esto supone la entrada de accionistas nuevos, por lo que suelen depender de los recursos propios, y sólo acudirán al endeudamiento, como ya se ha comentado, en caso de que los fondos propios no sean suficientes (Rodríguez y López, 2004).

A partir de la revisión de la literatura realizada sobre la relación entre el endeudamiento y la empresa familiar, en este trabajo se pretende aportar resultados y conclusiones al respecto, así se propone la siguiente hipótesis:

Hipótesis 2: Existe una relación negativa entre la decisión de exportar de las empresas familiares y su nivel de endeudamiento. 
El fenómeno de la internacionalización ha sido ampliamente estudiado en la literatura, siendo el tamaño de la empresa una de las variables analizadas. Así numerosos trabajos han estudiado la relación entre tamaño empresarial y exportación (ie., Alonso y Donoso, 1994; Barroso et al., 1998; Bonacorsi, 1992; Calof, 1994; Wagner, 2001). La mayoría de los trabajos empíricos encuentran una relación positiva entre el tamaño de la empresa y la actividad exportadora, lo cual se sustenta con argumentos teóricos evidentes, ya que las empresas pequeñas, en general, cuentan con menos recursos de todo tipo para poder iniciar este proceso (Manolova et al., 2002); sin embargo, otros estudios sostienen que el tamaño empresarial no siempre determina la actividad internacional de la empresa (Majocchi y Zucchella, 2003). En el caso de las empresas familiares, diferentes trabajos muestran que, en general, son de menor tamaño que las no familiares (Anderson et al., 2003; Claver et al., 2006).

Por otra parte, y aunque no existe un amplio consenso en la literatura, la mayoría de los autores coincide en señalar que las empresa familiares de segunda o tercera generación, es decir, aquellas que llevan más años en el mercado, son más proclives a emprender un proceso de internacionalización (Gallo y García Pont, 1996; Fernández y Nieto, 2005; Okoroafo y Perryy 2010). Finalmente, de la misma manera que el resto de las empresas, las familiares podrían iniciar un proceso de diversificación con el objetivo de reducir el riesgo global de la compañía, mejorar su posición competitiva en el futuro, alcanzar una mayor estabilidad y lograr una mayor creación de valor (Haque y Hassan, 2001, Lester y Parnell, 2006). En este sentido, autores como Otto y Marjo-Riitta (1994) señalan el número de productos, entre otros factores, como una variable que influye en la decisión de exportar. Por este motivo, se han incluido en este trabajo las variables tamaño, antigüedad y número de productos de la empresa como variables de control.

\section{Datos y Metodología}

\subsection{La muestra y las fuentes de datos}

Para la elaboración de este estudio se han utilizado los datos procedentes de la Encuesta sobre Estrategias Empresariales (ESEE). La ESEE es una investigación estadística que encuesta anualmente a un panel de empresas españolas manufactureras sobre distintos aspectos relacionados con su comportamiento estratégico y decisiones, y que incorpora además, información sobre sus Cuentas de Resultados y Balances Contables. La muestra que se presenta es representativa del sector

\section{Tabla 1}

Ficha técnica del estudio.

\begin{tabular}{ll} 
Población & \\
\hline Unidad & Empresa manufacturera española \\
$\begin{array}{l}\text { Diseño del cuestionario } \\
\text { Tipo de población }\end{array}$ & $\begin{array}{l}\text { Fundación SEPI } \\
\text { Alcance }\end{array}$ \\
Periodo temporal 100.000 elementos. & Nacional \\
\hline & Datos del año 2007 \\
Muestreo & \\
\hline Tipo de muestreo & Aleatorio estratificado y censal según tamaño \\
& de empresa y sector de actividad. \\
Tamaño muestral & 1.846 empresas manufactureras españolas \\
& $0,02(\mathrm{p}=\mathrm{q}=0,50)$ \\
Error muestral & $95 \%$ K=2 sigma) \\
Nivel de confianza & Soluciones Estadísticas de Productos y \\
Tratamiento de la información & Servicios (SPSS) \\
\hline
\end{tabular}


manufacturero español. Este trabajo se centra en el año 2007, previo a la actual crisis económica, con el objeto de que los resultados no se vean afectados por decisiones de las empresas en tiempos de recesión. En la tabla 1 se presenta la ficha técnica del estudio realizado.

\subsection{Las variables y su medición}

En este trabajo se presentan distintos modelos comparativos, para empresas familiares y no familiares. Dichos modelos analizan la decisión exportadora de la empresa manufacturera española, tomando como variable dependiente la actividad exportadora.

La variable dependiente será una variable dicotómica que tomará valores 0 y 1 . Tomará valor 0 si no exporta y valor 1 en caso de que sí lo haga.

Como variable explicativa en el primer modelo se ha incluido el carácter familiar de la empresa (FAM). Se define como una variable dicotómica que toma valores 0,1 . Tomará valor 1 cuando la empresa sea familiar y valor 0 cuando no lo sea. Se intenta analizar así, si el carácter familiar de una empresa puede favorecer a la actividad internacional $o$ viceversa. Se pretende analizar con ello si el carácter familiar tiene una relación negativa con los procesos de internacionalización, como indica la literatura, y si el comportamiento de este tipo de empresas difiere o no de las no familiares.

\section{Tabla 2}

Variables de los modelos propuestos.

\begin{tabular}{|c|c|c|c|c|c|}
\hline $\begin{array}{l}\text { Tipo de } \\
\text { Variable }\end{array}$ & $\begin{array}{l}\text { Aspecto a } \\
\text { estudiar }\end{array}$ & $\begin{array}{l}\text { Variable a } \\
\text { analizar }\end{array}$ & Definición & Nombre & Valores \\
\hline Dependiente & $\begin{array}{l}\text { Decisión de } \\
\text { exportar de } \\
\text { la empresa }\end{array}$ & $\begin{array}{l}\text { Actividad } \\
\text { exportadora }\end{array}$ & $\begin{array}{l}\text { Analiza si la empresa } \\
\text { exporta o no exporta }\end{array}$ & EXP & $\begin{array}{l}0=\text { No exporta } \\
1=\text { Sí exporta }\end{array}$ \\
\hline \multirow[t]{4}{*}{ Independientes } & $\begin{array}{l}\text { Dependencia } \\
\text { financiera }\end{array}$ & $\begin{array}{l}\text { Ratio de } \\
\text { endeudamient } \\
\text { o }\end{array}$ & $\begin{array}{l}\text { Porcentaje de recursos } \\
\text { ajenos sobre el total de } \\
\text { financiación que tiene la } \\
\text { empresa }\end{array}$ & DEU & Continua (\%) \\
\hline & $\begin{array}{l}\text { Carácter } \\
\text { familiar }\end{array}$ & $\begin{array}{l}\text { Gestión } \\
\text { Familiar }\end{array}$ & $\begin{array}{l}\text { Si la propiedad de la } \\
\text { empresa es familiar o no lo } \\
\text { es }\end{array}$ & FAM. & $\begin{array}{l}0=\text { No es } \\
\text { familiar } \\
1=\text { Sí es } \\
\text { familiar }\end{array}$ \\
\hline & $\begin{array}{l}\text { Variable de } \\
\text { interacción: } \\
\text { Endeudamie } \\
\text { nto ajustado } \\
\text { por el } \\
\text { carácter } \\
\text { familiar }\end{array}$ & $\begin{array}{l}\text { Ratio de } \\
\text { endeudamient } \\
\text { o*carácter } \\
\text { familiar }\end{array}$ & $\begin{array}{l}\text { Porcentaje de recursos } \\
\text { ajenos sobre el total de } \\
\text { financiación que tiene la } \\
\text { empresa ajustado por el } \\
\text { carácter familiar de la } \\
\text { empresa }\end{array}$ & $\begin{array}{l}\text { DEU_FA } \\
M\end{array}$ & Continua (\%) \\
\hline & $\begin{array}{l}\text { Estrategias } \\
\text { de } \\
\text { diversificaci } \\
\text { ón }\end{array}$ & $\begin{array}{l}\text { Diversificació } \\
\mathrm{n}\end{array}$ & $\begin{array}{l}\text { Oferta de productos o } \\
\text { servicios pertenecientes a } \\
\text { distintos sectores de } \\
\text { actividad. }\end{array}$ & DIV. & $\begin{array}{l}0=\text { No } \\
\text { diversifica } \\
1=\text { Sí } \\
\text { diversifica }\end{array}$ \\
\hline \multirow[t]{2}{*}{ De control } & Tamaño & $\begin{array}{l}\text { Número de } \\
\text { Trabajadores }\end{array}$ & $\begin{array}{l}\text { Número de trabajadores en } \\
\text { la empresa. }\end{array}$ & TAM. & Continua \\
\hline & $\begin{array}{l}\text { Antigüedad } \\
\text { de la } \\
\text { empresa }\end{array}$ & Años & $\begin{array}{l}\text { Años que la empresa lleva } \\
\text { actuando en el mercado } \\
\text { según la fecha de } \\
\text { constitución de la misma. }\end{array}$ & ANT. & $\begin{array}{l}0=\text { Más de } 50 \\
1=\text { Entre } 20-50 \\
\text { años. } \\
2=\text { Menos de } \\
20 \text { años. }\end{array}$ \\
\hline Dummy & $\begin{array}{l}\text { Sector de } \\
\text { actividad }\end{array}$ & $\begin{array}{l}\text { Sector de } \\
\text { actividad }\end{array}$ & $\begin{array}{l}\text { Sector en el que realiza la } \\
\text { empresa su actividad. }\end{array}$ & ACT. & $\begin{array}{l}\text { Véase tabla } 3 \\
\text { y } 4 .\end{array}$ \\
\hline
\end{tabular}


Tabla 3

Valores y frecuencias de los sectores de actividad.

\begin{tabular}{llll}
\hline Valor & industria & Frecuencia & Porcentaje \\
\hline 0 & Otras industrias manufactureras & 32 & 1,7 \\
1 & Industria cárnica & 60 & 3,2 \\
2 & Productos alimenticios y tabaco & 172 & 9,3 \\
3 & Bebidas & 36 & 1,9 \\
4 & Textiles y vestido & 128 & 6,9 \\
5 & Cuero y calzado & 42 & 2,3 \\
6 & Industria de la madera & 65 & 3,5 \\
7 & Industria del papel & 62 & 3,4 \\
8 & Edición y artes gráficas & 102 & 5,5 \\
9 & Productos químicos & 120 & 6,5 \\
10 & Productos de caucho y plástico & 99 & 5,4 \\
11 & Productos minerales no metálicos & 145 & 7,8 \\
12 & Metales férreos y no férreos & 66 & 3,6 \\
13 & Productos metálicos & 235 & 12,7 \\
14 & Máquinas agrícolas e industriales & 127 & 6,9 \\
15 & Máquinas de oficina, datos... & 23 & 1,2 \\
16 & Maquinaria y material eléctrico & 107 & 5,8 \\
17 & Vehículos de motor & 92 & 5,0 \\
18 & Otro material de trasporte & 36 & 1,9 \\
19 & Industria del mueble & 97 & 5,2 \\
\hline & $\quad$ Total & 1846 & 99,8 \\
Perdidos Sistema & 4 & 0,2 \\
\hline Total & $\quad 1850$ & 100,0 \\
\hline & & &
\end{tabular}

Tabla 4

Agrupación de sectores para tratamiento de datos.

\begin{tabular}{llll}
\hline Denominación & Definición & Dígitos & Valores \\
\hline ALI & Alimentación, bebidas y tabaco & $0,1,2$ y 3 & $\begin{array}{l}0=\text { Pertenece al sector } \\
\text { 1=No pertenece al sector }\end{array}$ \\
TEX.OTR & Textil, calzados, cuero y otros & $4,5,8$ y 19 & $0=$ Pertenece al sector \\
QUI.M & Productos químicos, minerales y de & $6,7,9,10,11,12,13$ & $1=$ No pertenece al sector \\
& la madera. & y 18 & \\
MAQ.TR & Maquinaria y transportes & $14,15,16,17$ & $0=$ Pertenece al sector \\
\hline
\end{tabular}

empresas familiares y su nivel de endeudamiento. En el presente trabajo, se va a analizar si esta variable influye en la actividad

El endeudamiento de la empresa (DEU), medido por el porcentaje de recursos ajenos entre el total de pasivo, es un ratio que explica en qué medida la empresa puede financiar su actividad con sus propios recursos y qué grado de dependencia tiene con agentes externos. Como se ha expuesto en la revisión de la literatura, existen evidencias sobre la relación negativa entre la actividad exportadora de las exportadora de la empresa y en su caso, en el proceso de internacionalización que pueda estar llevando a cabo, así como las diferencias que pueda haber en la relación de estas variables con las empresas no familiares.

$$
\mathrm{DEU}=(\text { Recursos ajenos/Pasivo total }) * 100
$$

Se ha añadido también al modelo una variable de interacción: DEU_FAM. El objetivo 
es poder analizar si el efecto de la deuda realmente es mayor en las empresas por su carácter familiar. Así ésta variable se define:

DEU_FAM $=\quad($ Recursos ajenos/Pasivo total $) *$ Carácter familiar $* 100$

La diversificación es otra de las variables que se han incluido en los modelos que se presentan (DIV). Esto se debe a que una de las razones que llevan a una empresa a exportar, es intentar conseguir una reducción de su riesgo global, de la misma manera que se puede conseguir con una estrategia de diversificación. Se ha incluido como variable dicotómica que toma dos valores 0,1 . Toma valor 1 cuando la empresa diversifica y 0 cuando no lo hace. La empresa no está diversificada si sólo define un producto a 3 dígitos de códigos CNAE y sí diversifica si define más de un producto en distintos códigos, del mismo sector (relacionada) o de distinto sector (no relacionada) (Almodóvar et al., 2009).

La edad de la empresa es un factor que también puede afectar a su proyección internacional (ANT). La literatura confirma que las empresas jóvenes tienen una menor orientación internacional que las más antiguas. Por otro lado, el desarrollo en los mercados internacionales puede llevar tiempo, por lo que aquellas de mayor edad tendrán una mayor penetración en dichos mercados (Smith et al., 2002). De este modo, se ha creado una variable que indica la antigüedad de la empresa. Esta variable toma valores 0,1 y 2 , según si la empresa tiene más de 50 años, entre 20 y 50 o menos de 20 años respectivamente. Asimismo, se ha incluido el tamaño de la empresa medido por el número de trabajadores (TAM)..
El tamaño ha sido una de las variables más ampliamente analizadas en la literatura empírica sobre el comportamiento exportador de la empresa (Alonso y Donoso, 1994; Barroso et al., 1998; Bonacorsi, 1992; Calof, 1994). Se considera que aquellas empresas de mayor tamaño tienen una mayor disponibilidad de recursos para iniciar otras actividades. La evidencia generalizada demuestra que un mayor tamaño incrementa la probabilidad de que una empresa exporte y también, aunque el consenso es menor, lo hace la intensidad exportadora (Bonaccorsi, 1992; Wagner, 2001). Por lo tanto, se incluye una variable de control que es el tamaño de la empresa, definida como una variable continua y medida por el número de empleados de la misma.

Por último, el sector en el que una empresa interactúa puede condicionar, de algún modo, su estrategia y sus resultados. Así, se incluye una variable dummy para identificar la pertenencia de la empresa a un determinado sector de actividad (López, 2006; Almodóvar et al., 2009). En la tabla 2 se describen todas las variables, con su nomenclatura y medición.

\subsection{Metodología de análisis}

El estudio que se expone a continuación presenta varios análisis para los cuales se ha empleado un modelo de regresión logística binaria, modelo Logit. Este tipo de regresión resulta adecuada, dada la naturaleza de la variable dependiente que se está midiendo en este caso, ya que se trata de una variable limitada, dicotómica, por lo que las técnicas de regresión lineal no resultan adecuadas, debido a que los valores ajustados de una regresión lineal

\section{Tabla 5}

Modelos para el análisis.

\begin{tabular}{|c|c|c|c|}
\hline Modelos & Objeto de análisis & $\begin{array}{l}\text { Variable } \\
\text { depediente }\end{array}$ & $\begin{array}{l}\text { Tamaño } \\
\text { de la } \\
\text { muestra }\end{array}$ \\
\hline Modelo 1a & Empresas manufactureras españolas & $\begin{array}{l}\text { Dicotómica: } \\
\text { Exportación }\end{array}$ & 1.846 \\
\hline Modelo $1 \mathrm{~b}$ & Empresas manufactureras españolas no familiares & $\begin{array}{l}\text { Dicotómica: } \\
\text { Exportación }\end{array}$ & 1.136 \\
\hline Modelo 1c & Empresas manufactureras españolas familiares & $\begin{array}{l}\text { Dicotómica: } \\
\text { Exportación }\end{array}$ & 710 \\
\hline
\end{tabular}




\section{Tabla 6}

Estadísticos descriptivos.

\begin{tabular}{|c|c|c|c|c|c|c|}
\hline \multirow[t]{2}{*}{ Variables del modelo } & \multicolumn{2}{|l|}{ Modelo 1 a } & \multicolumn{2}{|c|}{ Modelo $1 \mathrm{~b}$} & \multicolumn{2}{|c|}{ Modelo $1 \mathrm{c}$} \\
\hline & $\begin{array}{l}\text { Media } \\
\text { (Máx.- } \\
\text { Mín.) }\end{array}$ & Desv. típ. & $\begin{array}{l}\text { Media } \\
\text { (Máx.- } \\
\text { Mín.) }\end{array}$ & Desv. típ. & $\begin{array}{l}\text { Media } \\
\text { (Máx.- } \\
\text { Mín.) }\end{array}$ & Desv. típ. \\
\hline EXP & $\begin{array}{l}0,62 \\
(0-1)\end{array}$ & 0,485 & $\begin{array}{l}0,61 \\
(0-1)\end{array}$ & 0,488 & $\begin{array}{l}0,63 \\
(0-1)\end{array}$ & 0,483 \\
\hline ANT & $\begin{array}{l}1,36 \\
(0-2)\end{array}$ & 0,699 & $\begin{array}{l}1,37 \\
(0-2)\end{array}$ & 0,711 & $\begin{array}{l}1,35 \\
(0-2)\end{array}$ & 0,677 \\
\hline FAM & $\begin{array}{l}0,38 \\
(0-1)\end{array}$ & 0,487 & & & & \\
\hline DEU & $\begin{array}{l}57,5599 \\
(2-99,9)\end{array}$ & 22,77242 & $\begin{array}{l}\mathbf{5 8 , 5 2 8 2} \\
(2-99,9)\end{array}$ & 22,68463 & $\begin{array}{l}\mathbf{5 6 , 0 2 3 2} \\
(2,9- \\
99,5)\end{array}$ & 22,84331 \\
\hline DEU_FAM & $\begin{array}{l}20,95 \\
(0-100)\end{array}$ & 30,499 & & & & \\
\hline DIV & $\begin{array}{l}0,15 \\
(0-1)\end{array}$ & 0,357 & $\begin{array}{l}0,16 \\
(0-1)\end{array}$ & 0,363 & $\begin{array}{l}0,14 \\
(0-1)\end{array}$ & 0,346 \\
\hline TAM & $\begin{array}{l}243,27 \\
(1-13.290)\end{array}$ & 725,13 & $\begin{array}{l}293 \\
(1- \\
13.290)\end{array}$ & 857,223 & $\begin{array}{l}\mathbf{1 6 3 , 6 7} \\
(3- \\
7.295)\end{array}$ & 426,588 \\
\hline QUI.M & $\begin{array}{l}0,45 \\
(0-1)\end{array}$ & 0,498 & $\begin{array}{l}0,46 \\
(0-1)\end{array}$ & 0,499 & $\begin{array}{l}0,44 \\
(0-1)\end{array}$ & 0,496 \\
\hline MAQ.TRA & $\begin{array}{l}0,19 \\
(0-1)\end{array}$ & 0,392 & $\begin{array}{l}0,21 \\
(0-1)\end{array}$ & 0,407 & $\begin{array}{l}0,16 \\
(0-1)\end{array}$ & 0,365 \\
\hline TEX.OTR & $\begin{array}{l}0,20 \\
(0-1)\end{array}$ & 0,400 & $\begin{array}{l}0,19 \\
(0-1)\end{array}$ & 0,394 & $\begin{array}{l}0,21 \\
(0-1)\end{array}$ & 0,409 \\
\hline ALI & $\begin{array}{l}0,16 \\
(0-1)\end{array}$ & 0,369 & $\begin{array}{l}0,14 \\
(0-1)\end{array}$ & 0,346 & $\begin{array}{l}0,20 \\
(0-1)\end{array}$ & 0,400 \\
\hline
\end{tabular}

Tabla 7

Coeficientes de correlación entre variables independientes.

\begin{tabular}{ccccccccc}
\hline & ANT & FAM & DEU & QUI.M & MAQ.TRA & TEX.OTR & DIV & TAM \\
\hline ANT & 1 & & & & & & & \\
FAM & - & 1 & & & & & & \\
& 0,018 & & & & & & & \\
DEU & 0,186 & - & 1 & & & & & \\
& & 0,049 & & & & & & \\
QUI.M & 0,044 & - & 0,056 & 1 & & & & \\
& & 0,028 & & & & & & \\
MAQ.TRA & - & - & $-0,036$ & $-0,439$ & 1 & & & \\
TEX.OTR & 0,052 & 0,063 & & & & & & \\
DIV & - & $-0,025$ & $-0,020$ & $-0,455$ & $-0,241$ & 1 & \\
& 0,058 & 0,024 & $-0,016$ & 0,027 & 0,085 & $-0,065$ & 1 & \\
TAM & - & - & 0,013 & 0,026 & 0,139 & $-0,182$ & 0,089 & 1
\end{tabular}

Para cada par de variables continuas se reporta el coef. de corr. de Pearson; en caso contrario se reporta el coef. de corr. no paramétrica de Spearman.

no tienen la restricción de estar situados entre cero y uno. En la tabla 5 se exponen los modelos con sus características.

\section{Resultados}

En la tabla 6 se recogen los estadísticos descriptivos de las variables de las distintas muestras. 
Como puede observarse, destacan dos variables: la primera es el tamaño empresarial. Como se exponía en la revisión bibliográfica, las empresas familiares tienen en global un menor tamaño que las empresas no familiares, lo cual puede explicar su menor propensión exportadora.

En segundo lugar, el nivel de endeudamiento de las empresas no familiares es ligeramente menor que el de las empresas familiares, no obstante es tan irrelevante la diferencia que es necesario un análisis posterior más exhaustivo desarrollado con otras técnicas.

En la tabla 7 se exponen los coeficientes de correlación de Spearman y Pearson entre las variables independientes.

Inicialmente, se ha hecho un test de igualdad de medias entre empresas familiares y no familiares con las variables dependientes y variables independientes (Tabla 8).

Los resultados muestran que el nivel de endeudamiento presenta una diferencia significativa entre ambos tipos de empresas. Estos resultados pueden dar algunos indicios a las hipótesis planteadas que para poder contrastarlas definitivamente. Por este motivo, es interesante ampliar este análisis con modelos econométricos posteriores.

\section{Tabla 8}

Test de igualdad de medias entre empresas familiares y no familiares.

\begin{tabular}{cc}
\hline & Diferencia de medias \\
\hline EXP & 0,23 \\
ANT & 0,13 \\
TAM. & $0,17^{* * *}$ \\
DIV & 0,018 \\
DEU & $\mathbf{2 , 5 0 * *}$ \\
\hline
\end{tabular}

${ }^{* *} \mathrm{p}<0.05 ; * * * \mathrm{p}<0.01$

En la tabla 9 se detallan los resultados de los distintos modelos. Puede observarse que las variables de control: tamaño y antigüedad de la empresa, son significativas en los análisis realizados, lo cual aporta robustez a los modelos propuestos. En cuanto a las variables que ocupan el estudio, cabe destacar, que la propiedad familiar de las empresas no es un factor significativo para la toma de decisión sobre la exportación o no de la empresa $\left(\beta_{\mathrm{FAM}}=\right.$ $0.119)$. En este sentido no podemos aceptar la hipótesis 1 propuesta.

También se puede observar que en todos los modelos propuestos, las empresas que pertenecen al sector de maquinaria y transportes son más influyentes en la exportación que las empresas de otros sectores como el de la alimentación, bebidas y tabaco.

Con respecto a los factores que influyen en la decisión de exportar, hay que señalar que en el modelo 1, tanto el tipo 1a como el tipo 1b, explican mejor el comportamiento exportador de las empresas no familiares que el de las empresas de propiedad familiar; véase las respectivas $\mathrm{R}$ cuadrado, las cuales son mayores en la muestra de empresas no familiares que en el de las familiares. Si bien es cierto que $R$ cuadrado no son muy altas $\left(\mathrm{R}_{1 \mathrm{a}}^{2}=0.19, \mathrm{R}_{1 \mathrm{~b}}{ }^{2}=0.23\right.$ y $\left.\mathrm{R}_{1 \mathrm{c}}^{2}=0.13\right)$, están en línea con otros trabajos, como López (2006) y Robson y Bennett (2000), además los resultados de los porcentajes de acierto de los modelos son aceptables como puede observarse.

Igualmente, se puede observar cómo la diversificación es un factor explicativo $\left(\beta_{\mathrm{DIV}}=0.302 *\right)$. Esto puede deberse a que la diversificación exige una serie de cambios que supone romper con la situación presente de la empresa. La empresa familiar no siempre está dispuesta a llevar a cabo políticas que impliquen la ruptura de la tradición. Éstas, caracterizadas por la aversión a los cambios que impliquen riesgos, como ya se ha puesto de manifiesto, pueden no ver en la diversificación una estrategia de disminución del riesgo global, sino todo lo contrario.

No obstante, los resultados más interesantes desde el punto de vista de la segunda hipótesis formulada se obtienen en el modelo 1a. Dicho modelo se ha estimado con la variable DEU y, posteriormente, con la variable interacción. La primera estimación da indicios de que esta variable es influyente a la hora de que una empresa se decida por exportar y ampliar negocio en el extranjero $\left(\beta_{\mathrm{DEB}}=-0.006^{* *}\right)$, sin embargo, no se puede deducir que sea por el carácter familiar de la misma. En la segunda 


\section{Tabla 9}

Análisis logístico binario. Variable dependiente EXP.

\begin{tabular}{|c|c|c|c|c|c|c|c|c|}
\hline \multirow[b]{3}{*}{$\begin{array}{c}\text { Variables } \\
\text { independientes }\end{array}$} & \multicolumn{4}{|c|}{ Modelo 1a. Total empresas } & \multirow{2}{*}{\multicolumn{2}{|c|}{$\begin{array}{c}\text { Modelo } 1 \mathrm{~b} \\
\text { Empresas no familiares }\end{array}$}} & \multirow{2}{*}{\multicolumn{2}{|c|}{$\begin{array}{c}\text { Modelo } 1 c \\
\text { Empresas familiares }\end{array}$}} \\
\hline & \multicolumn{2}{|c|}{ Con variable DEU } & \multicolumn{2}{|c|}{$\begin{array}{l}\text { Introduciendo variable } \\
\text { interacción DEU FAM }\end{array}$} & & & & \\
\hline & Coeficiente & $\begin{array}{c}\text { Error } \\
\text { Estándar }\end{array}$ & Coeficiente & $\begin{array}{c}\text { Error } \\
\text { Estándar }\end{array}$ & Coeficiente & $\begin{array}{c}\text { Error } \\
\text { Estándar }\end{array}$ & Coeficiente & $\begin{array}{c}\text { Error } \\
\text { Estándar }\end{array}$ \\
\hline ANT & $-0,327 * * *$ & 0,087 & $-0,324 * * *$ & 0,087 & $-0,256 * *$ & 0,114 & $-0,438 * *$ & 0,140 \\
\hline FAM & 0,119 & 0,111 & $0,632 * *$ & 0,302 & & & & \\
\hline DEU & $-0,006^{* *}$ & 0,002 & $-0,003$ & 0,003 & $-0,003$ & 0,003 & $-0,010 * *$ & 0,004 \\
\hline DEU_FAM & & & $-0,009 *$ & 0,005 & & & & \\
\hline DIV & $0,302 *$ & 0,161 & $0,303 *$ & 0,161 & 0,327 & 0,210 & 0,285 & 0,255 \\
\hline TAM & $0,005 * * *$ & 0,000 & $0,005 * * *$ & 0,000 & $0,007 * * *$ & 0,001 & $0,003 * * *$ & 0,001 \\
\hline TEX.OTR & 0,126 & 0,176 & 0,140 & 0,177 & 0,169 & 0,245 & 0,179 & 0,262 \\
\hline QUI.M & 0,060 & 0,156 & 0,079 & 0,156 & 0,346 & 0,217 & $-0,261$ & 0,229 \\
\hline MAQ.TR & $0,941 * * *$ & 0,199 & $0,955 * * *$ & 0,199 & $1,061 * * *$ & 0,265 & $0,902 * * *$ & 0,313 \\
\hline CTE. & 0,395 & 0,231 & 0,165 & 0,262 & $-0,191$ & 0,314 & $1,178 * * *$ & 0,322 \\
\hline $\begin{array}{l}\mathrm{R}^{2} \text { de Cox y } \\
\text { Snell }\end{array}$ & 0,18 & & 0,19 & & 0,23 & & 0,1 & \\
\hline $\begin{array}{l}\text { Porcentaje de } \\
\text { aciertos del } \\
\text { modelo }\end{array}$ & 72.6 & & 73.0 & & 74.8 & & 67 & \\
\hline
\end{tabular}

$* \mathrm{p}<0.1 ; * \mathrm{p}<0.05 ; * * * \mathrm{p}<0.01$

estimación del modelo, se ha introducido la variable de interacción: DEU FAM, la cual se muestra significativa $\left(\beta_{\mathrm{FAM} * \mathrm{DEB}}=-0.009 *\right)$. Gracias a esta variable de interacción sí puede deducirse que el efecto de la deuda es mayor en las empresas familiares que en las no familiares a la hora de exportar.

A estos resultados hay que añadir los obtenidos en el modelo $1 \mathrm{~b}$ y $1 \mathrm{c}$ ya que los corroboran $\left(\beta_{\text {DEB } \_1 b}=-0.003, \beta_{\text {DEB }_{-} 1 c^{1}}=-0.010^{* *}\right)$. En este análisis se pone de manifiesto que el endeudamiento es significativo para las empresas familiares, al contrario que para las empresas no familiares a la hora de tomar decisiones de exportación. Esto se puede deber a que las empresas no familiares no condicionan su actividad exportadora por el hecho de endeudarse, sin embargo, las familiares sí lo hacen. Como ya se apuntó en la revisión de la literatura, este tipo de empresas prefiere acudir a las fuentes de financiación propia, antes que al endeudamiento para financiar sus inversiones y sus procesos de expansión y cambio. Por otra parte, puede verse que la relación entre las variables exportación y endeudamiento es negativa. En este sentido se puede aceptar la hipótesis 2 propuesta.

\section{Conclusiones}

Los resultados obtenidos parecen concluir que el carácter familiar de una empresa no influye en su decisión de exportar. Así mismo, de los resultados se desprende que ciertos factores, cuya influencia sobre las empresas ha sido contrastada por la literatura desde un punto de vista general, pierden poder a la hora de explicar la decisión de exportar en el caso de las empresas con propiedad familiar.

Las dificultades asociadas a la toma de decisiones de exportación de la empresa familiar pueden ser mayores si no se dispone de una holgada financiación interna para abordar el proceso. En este caso, la solución pasa por encontrar financiación externa, mediante deuda o bien mediante ampliaciones de capital. La literatura demuestra que las empresas familiares 
no suelen acudir a las ampliaciones de capital, si eso supone la entrada de accionistas no familiares y la consiguiente pérdida de control de la compañía por parte de la familia. Así pues, la otra opción posible es acudir a la financiación ajena; sin embargo, los resultados del modelo muestran que las empresas familiares intentan endeudarse lo menos posible, optando en ocasiones por no llevar a cabo la estrategia de exportación si por ello tienen que incrementar su dependencia financiera, dado que esto supone una entrada de acreedores en el pasivo del balance. Esto produce efectos negativos en la actividad exportadora, puesto que, como puede observarse en los resultados, ambas variables: endeudamiento y exportación, se relacionan de forma significativa con signo negativo.

Igualmente, atendiendo a los distintos factores que han explicado tradicionalmente la exportación de las empresas, como puede ser la diversificación, la cual siempre ha tenido un papel positivo en el fomento de la exportación en general, no se corresponde con los resultados obtenidos para las empresas familiares, ya que este factor se muestra como no significativo. Por su parte, otros factores también estudiados a lo largo de la historia empresarial, como el tamaño, la antigüedad y determinados sectores de actividad, sí contrastan sobradamente la relación positiva que mantienen con la actividad exportadora, tanto para las empresas familiares como para las no familiares.

Una de las conclusiones más significativas de la investigación realizada está relacionada con el ratio de endeudamiento, la estructura de capital de la empresa familiar y la actividad exportadora de la empresa. En este sentido, el endeudamiento es un factor influyente en su decisión de exportar. La internacionalización lleva asociado un importante grado de incertidumbre para una empresa normalmente pequeña, centrada en un mercado nacional (e incluso local), con un equipo directivo más bien conservador, reacio a asumir riesgos, y en las que se integran una cultura y unos valores tradicionales. Por ello, las motivaciones para iniciar este proceso van más allá de la capacidad de endeudamiento de la empresa, cobrando fuerza otros factores que no han sido objeto de análisis en el presente trabajo como son las ventajas competitivas, las alianzas estratégicas, etc.

En conclusión, algunos factores que explican la internacionalización de la empresa en general, no coinciden con los que influyen en la toma de decisiones de la empresa familiar, véase el caso de la diversificación. Mientras que otros factores tradicionalmente estudiados, como el tamaño, la actividad o la antigüedad, sí se mantienen. No obstante, uno de los principales obstáculos que hace que la empresa familiar exporte en menor medida que la empresa no familiar, es la necesidad de financiación que requieren las inversiones para llevar a cabo un proceso de este tipo. En este punto, el objetivo de crecimiento mediante la internacionalización de la empresa familiar se enfrenta a los intereses y demandas de la familia; máxime cuando dicha decisión supone arriesgar la riqueza de la misma y su medio de vida, por lo que suelen ser inversores más conservadores y cautos a la hora de tomar este tipo de decisiones.

Es necesario señalar que este conflicto de intereses perjudica el valor y el futuro desarrollo de la empresa familiar, ya que debido al ambiente externo altamente complejo y cambiante en que nos encontramos actualmente, es preciso alcanzar los parámetros de competitividad y renovación estratégica que el entorno impone para asegurar su supervivencia y continuidad.

En definitiva, los resultados obtenidos clarifican la práctica empresarial de las empresas familiares y pueden tener implicaciones a la hora de implementar políticas de impulso a la actividad exportadora y futura internacionalización de este tipo de empresas con el fin de garantizar su continuidad, especialmente en momentos económicos adversos como el que atraviesa España en la actualidad.

\section{Bibliografía}

Almodóvar, P., Navas, J. E. y Huerta, P. (2009). La tipología estratégica como factor determinante de la empresa conjunta internacional. Investigaciones Económicas, 33(3), 407-438. 
Alonso, J. A. y Donoso, V. (1994). Competitividad de la empresa exportadora española. Madrid: Editorial ICEX.

Anderson, R., Mansi, S. y Reeb, D. (2003). Founding family ownership and the agency cost ofdebt. Journal of Financial Economics, 68(2), 263285.

Aronoff, C. y Astrachan, J. (1996). Reducing the risks of family-business growth. Nation's Business, 84 (3), 52-53.

Barroso, C., Casillas, J. C. y Galan, J. L. (1998). Tamaño y rendimiento de las empresas exportadoras. AEDEM, Benalmádena, 61-69.

Barry, B. (1975). The Development of Organization Structure in the Family Firm. Journal of General Management, 3(1), 42-60.

Blanco, V., De Quevedo, E. y Delgado, J. (2009). La estructura financiera de la empresa familiar y el cambio generacional. Revista española de financiación y contabilidad, 38(141), 57-73.

Bonacorsi, A. (1992). On the relationship between firm size and export intensity. Journal of International Business Studies, 23(4), 605-35.

Calof, J. (1994). The relationship between firm size and export behavior revisited. Journal of International Business Studies, 25(2), 367-387.

Casillas, J.C. y Acedo, F.J. (2005). Internationalisation of Spanish Family SMEs: An analysis of Family Involvement. International Journal of Globalisation and Small Business, 1(2), 134-151.

Chaganti, R. y Damanpour, F. (1991). Institutional ownership, capital structure and firm performance. Strategic Management Journal, 12(7), 479-491.

Claver, E., Rienda, L. y Quer, D. (2006). El comportamiento de las empresas familiares y no familiares en los mercados extranjeros: un estudio comparativo. Cuadernos de Gestión, 6(2), 11-25.

Claver, E., Rienda, L. y Quer, D. (2009). Family Firms' International Commitment: The Influence of Family-Related Factors. Family Business Review, 22 (2), 125-135.

Corbetta, G. (1995). Patterns of development of family businesses in Italy. Family Business Review, 8(4), 255-265.

Davis, P. y Harveston, P. (2000). Internationalization and organizational growth: the impact of Internet Usage and Technology involvement among entrepreneur-led family businesses. Family Business Review, 13(2), 107-120.

Fernández, Z. y Nieto, M.J. (2005). Internationalization Strategy of Small and MediumSized Family Businesses: Some Influential Factors. Family Business Review, 18(1), 77-89.

Fuentes, G., Vallejo, M. y Fernández, R. (2007). Factores a tener en cuenta en la expansión de la empresa familiar. Investigaciones Europeas de Dirección y Economía de la Empresa, 13(2), 75-96.

Fuentes, G., Vallejo, M. y Fernández, R. (2007). Aspectos determinantes en la internacionalización de la empresa familiar. Revista de Estudios Empresariales, 1, 38-54.

Fuentes, G. y Fernández, R. (2010). Strategic alliances in the internationalization of family firms: An exploratory study on the Spanish wine industry. Advances in Management, 3(6), 45-54.

Gallo, M.A. y Sveen, J. (1991). Internationalizing the family business: facilitating and restraining factors. Family Business Review, 4(2), 181-190.

Gallo, M.A. y García-Pont, C. (1996). Important factors in family business internationalization. Family Business Review, 9(1), 45-59.

Gallo, M.A., Tàpies, J. and Cappuyns, K. (2004). Comparison of family and nonfamily business: Financiallogic and personal preferences. Family Business Review, 17(4), 303-318.

Gallo, M.A., Klein, S., Tomaselli, S., Montermerlo, D. and Cappuyns, K. (2009). La empresa familiar multigeneracional. Navarra: EUNSA.

Graves, C., y Thomas, J. (2008). Determinants of the Internationalization Pathways of Family Firms: An Examination on family business. Family Business Review, 21(2), 151-167.

Habbershon, T.G. y Williams, M.L. (2000). A model for understanding the competitiveness of family-controlled companies. In P. Poutziouris (ed) Tradition or entrepreneurship in the new economy. Manchester Business School, 94-115. Manchester.

Haque, M., y Hassan, M.K. (2001). Diversification as a corporate strategy for a familycontrolled business group in a frontier market. The Journal of Social, Political, and Economic Studies, 26(4), 719-758.

Hill, C., y Kim, W. (1990). Searching for a dynamic theory of the multinational enterprise: A 
transaction cost model. Strategic Management Journal, 11, 117-128.

Hutchinson, R. (1995). The capital structure and investment decisión of the small owner-managed firm: Some exploratory issues. Small Business Economics, 7(3), 231-239.

Johanson, J. and Vahlne, J. (1977). The internationalization process of the firm - a model of knowledge development and increasing foreing market commitments. Journal of International Business Studies, 8, 23-32.

Johanson, J. and Vahlne, J. (1990). The mechanism of internationalisation. International Marketing Review, 7(4), 11-24.

Johanson, J. and Wiedershein, F. (1975). The internationalization of the firm- four Swedish cases. Journal of Management Studies, 12(3), 305-22.

Karlsson, A. (2001). En famille: Invisible managers in owner families. Academic Research Forum Proceedings. FBN 12TH Annual World Conference Rome. University School of Management, Bocconi.

Lansberg, I. (1999). Succeeding generations: Realizing the dream of families in business. Boston, Ma: Harvard Business School Press.

Lester, D., y Parnell, J. (2006). The Complete Life Cycle of a Family Business. Journal of Applied Management and Entrepreneurship, 11(3), 475-487.

López, J. (2006). La internacionalización de la empresa manufacturera española: Efectos del capital humano genérico y específico. Cuadernos de Gestión, 6(1), 11-24.

López-Cózar, C. y Priede, T. (2009). Empresa familiar claves para su supervivencia en un mundo cambiante. La Coruña. Netbiblo.

López-Gracia, J. y Aybar-Arias, C. (2000). An empirical approach to the financial behaviour of small andmedium sized companies. Small Business Economics, 14(1), 55-63.

Luostarinem, R. (1970). Foreign operations of the firm. Helsinki: Helsinki School of Economics.

Luostarinem, R. y Hellman, H. (1994). The internationalization processes and strategies of Finnish family firms. CIBR Research Papers, Helsinki.

Lussier, R., y Sonfield, M. (2009). Founder influences in family businesses: analyzing combined data from six diverse countries. Journal of Small Business Strategy, 20(1), 103-118.
Majocchi, A. and Zucchella, A. 2003. Internationalization and Performance: Findings from a Set of Italian SMEs. International Small Business Journal, 21(3): 249-268.

Manolova, T., Brush, C., Edelman, L. and Greene, P. 2002. Internationalization of Small Firms: Personal Factors Revisited. International Small Business Journal, 20(1): 9-31.

Martín, J. (2004). Formas de entrada en los mercados exteriores. Boletín Económico del ICE, Información Comercial Española, 2823, 33-48.

Martín, J. y Cabrera, K. (2007). La gestión del marketing estratégico en la pequeña empresa familiar. Cuadernos de Gestión, 7(1), 85-100.

Menéndez, S. (2005). Growth and Internationalisation of Family Businesses. International Journal of Globalisation and Small Business, 1(2), 122-133.

Miller, D., Le Breton-Miller, I., y Lester, R. (2010). Family and Lone Founder Ownership and Strategic Behaviour: Social Context, Identity, and Institutional Logics. Journal of Management Studies, 48(1), 1-25.

Myers, S. y Majluf, S. (1984). Corporate financing and investment decisions when firms have information that investors do not have. Journal of Financial Economy, 13(1), 187-221.

Okoroafo, S.C. (1999). Internationalization of family businesses: Evidence from NorthwestOhio, USA. Famliy Business Review, 12, 147-158.

Okoroafo, S.C., y Perryy, M. (2010). Generational Perspectives of the Export Behavior of Family Businesses. International Journal of Economics and Finance, 2(3), 15-24.

Otto, A., y Marjo-Riitta, R. (1994). Prediction of export intentions - managing with structural characteristics? Scandinavian Journal of Management, 10(1), 17-27.

Pla-Barber, J. (2001). The internalisation of foreign distribution and production activities. New empirical evidence from Spain. International Business Review, 10, 455-474.

Poutziouris, P. (2001). The views of family companies on venture capital: Empirical evidence from the UK small to medium-size enterprising economy. Family Business Review, 14(3), 277-291.

Quintana, J. (2007). La internacionalización de la empresa familiar española. ICE, 839, 113-120. 
Robson, P., y Bennett, R. (2000). SME growth: The relationship with business advice and external collaboration. Small Business Economics, 15(3), 193-208.

Rodríguez, R., y López, M. (2004). La empresa familiar exportadora. El caso de las empresas de congelados y conservas de pescados, moluscos y crustáceos. Revista Galega de Economía, 13(1), 119.

Romano, C., Tanewski, G., y Smyrnios, K. (2000). Capital structure decision making: A model for family business. Journal of Business Venturing, 16 (3), 285-310.

Setia-Atmaja, L. (2010). Dividend and debt policies of family controlled firms; The impact of board independence. International Journal of Managerial Finance, 6(2), 128-142.

Sacristán, M., Rico, G. y Lafuente, C. (2011). La conducta exportadora de las Pymes y de las empresas familiares: ¿siguen una aproximación incremental? Revista de Empresa Familiar, (1) 1, 7 21.

Shu Ling Lin, y Ming Fung Wu (2010). Family ownership and risk-taking: Exploring nonlinear effects in financial industry. African Journal of Business Management, 4(17), 3738-3751.

Smith, V., Strojer, E., y Dilling-Hansen, M. (2002). Export performance and Investment in RyD, WP 2002/4. The Danish Institute for Studies in Research and Research Policy.

Sonfield, M., y Lussier, R. (2004). First-, Second-, and Third-Generation Family Firms: A Comparison. Family Business Review, 17(3), 189.

Swinth, R., y Vinton, K. (1993). Do familyowned business have a strategic advantage in international joint ventures? Family Business Review, 4(2), 19-30.

Vahlne, J., y Nordström, K. (1993). The internationalization process: impact of competition and experience. International Trade Journal, 7(5): 529-548.

Wagner, J. (2001). A note on the firm size-export relationship. Small Business Economics, 17, 229237.

Westhead, P., Wright, M., y Ucbasaran, D. (2001). Internationalization of New and Small Firms: A Resource-Based View. Journal of Business Venturing, 16, 333-358. 